\title{
Eliminação transepidérmica de parasitas na doença de Jorge Lobo
}

\section{Transepidermal elimination of parasites in Jorge Lobo's disease}

\author{
Mario F. R. Miranda ${ }^{1}$ \\ Maraya de Jesus S. Bittencourt ${ }^{3}$
}

\author{
Vivian S. da Costa ${ }^{2}$ \\ Arival C. de Brito ${ }^{4}$
}

\begin{abstract}
Resumo: Fundamentos: A eliminação transepidérmica de parasitas (ETEP) tem sido pouco estudada na doença de Jorge Lobo.

OBJETIVOS: Identificar aspectos morfológicos da ETEP na doença de Jorge Lobo.

MÉToDos: Recortes de biópsias de doença de Jorge Lobo emblocados em parafina foram corados pela hematoxilina-eosina e examinados. Considerou-se como ETEP, exclusivamente, a presença de parasitas em estruturas epidérmicas.

RESULTADOS: Foram incluídas no estudo 40 biópsias de 37 pacientes ( 31 homens e seis mulheres, média de idade 51,03 anos, variação 29-80 anos) realizadas em um período de 37 anos (1967-2003), das quais foram obtidos 511 cortes (média de 12,77 cortes por caso, variação 2-39 cortes por caso). Observou-se ETEP em 110/511 (21,52\%) e não se observou em 401/511 cortes $(78,48 \%)(p<0,0001)$. Em relação aos pacientes $(37)$, em 15 se verificaram aspectos consistentes com ETEP (40,5\%), ao passo que, em 22 deles $(59,5 \%)$, isso não foi observado ( $p>0,05)$. Os parasitas dispunham-se em infundíbulos hiperplásicos, formando catênulas, ou como unidades isoladas, associados ou não a células inflamatórias.

CONCLUSÕES: Aspectos consistentes com ETEP, embora observados em número estatisticamente não significante de pacientes da amostra $(p>0,05)$, sugerem que, na doença de Jorge Lobo, o fenômeno, invariavelmente, ocorra através do epitélio infundibular. Estudos futuros serão necessários para avaliar sua eventual importância na epidemiologia da micose.
\end{abstract}

Palavras-chave: Doença granulomatosa crônica; Micoses; Micoses/patologia; Pele

Abstiract: BACKGRound: Few studies have focussed on the transepidermal elimination of parasites in Jorge Lobo's disease (lobomycosis)

OвJестіve: To identify the morphological features of the transepidermal elimination of parasites in lobomycosis. METHODS: Sections were obtained from paraffin-embedded biopsy specimens of patients with lobomycosis and stained with hematoxylin-eosin for microscopic examination. Only the presence of parasites in epidermal structures was considered to constitute transepidermal elimination.

RESulTs: Forty biopsies from 37 patients were included in the study (31 males and 6 females). The mean age of patients was 51.03 years (range 29-80 years). Biopsies performed over a period of 37 years (1967-2003) were used, from which 511 sections were obtained (a mean of 12.77 sections per case; range 2-39 sections per case). Transepidermal elimination of parasites was found in 110/511 (21.52\%) and was absent in 401/511 sections $(78.48 \%)(\mathrm{p}<0.0001)$. Features consistent with the phenomenon were found in $15 / 37$ patients $(40.5 \%)$ and were absent in 22/37 (59.5\%) ( $p>0.05)$. Parasites in the epidermis were detected within hyperplastic infundibula, either connected in chains or as isolated units, associated or not with inflammatory cells.

CONCLUSION: Features consistent with transepidermal elimination of parasites were found in a statistically nonsignificant number of patients in the sample $(\mathrm{p}>0.05)$, suggesting that in Jorge Lobo's disease, this phenomenon invariably occurs through the infundibular epithelium. Future studies are required to evaluate the significance of this finding in the epidemiology of mycosis.

Key words: Lobomycosis; Jorge Lobo's disease; chronic granulomatous disease; mycoses; mycoses/pathology; skin.

Recebido em 22.04.2009.

Aprovado pelo Conselho Consultivo e aceito para publicação em 07.11.09.

Trabalho realizado no Serviço de Dermatologia do Instituto de Ciências da Saúde da Universidade Federal do Pará (ICS-UFPA), como parte do Projeto 3CCS010403C - "Integração Básico-Profissional na Formação Médica", coordenado pela Prof ${ }^{a}$ Dra. Réia Sílvia Lemos. Belém (PA), Brasil.

Conflito de interesse: Nenhum / Conflict of interest: None

Suporte financeiro / Financial funding: Oriundo do Projeto 3CCS010403C do Instituto de Ciências da Saúde (ex-Centro de Ciências da Saúde) da Universidade Federal do Pará (UFPA), exclusivamente sob a forma de reposição de material de consumo histológico.

Professor adjunto IV de Dermatologia da Universidade Federal do Pará (UFPA); responsável pelo laboratório de dermatopatologia do Serviço de Dermatologia do Instituto de Ciências da Saúde da Universidade Federal do Pará (ICS-UFPA) - Belém (PA), Brasil.

Estudante de graduação do curso de Medicina do Instituto de Ciências da Saúde da Universidade Federal do Pará (ICS-UFPA); participante do Projeto 3CCS010403C - Belém (PA), Brasil.

Médica dermatologista; ex-residente de Dermatologia do Hospital da Fundação Santa Casa de Misericórdia do Pará (HFSCMP)/ICS - Universidade Federal do Pará (UFPA) - Belém (PA), Brasil.

Doutor e livre-docente em Dermatologia da Universidade Federal do Pará (UFPA); professor do Programa de Pós-graduação em Doenças Tropicais do Instituto de Medicina Tropical da Universidade Federal do Pará (UFPA); professor do Programa de Pós-graduação em Biologia dos Agentes Infecciosos e Parasitários do Instituto de Ciências Biológicas da Universidade Federal do Pará (UFPA) - Belém (PA), Brasil. 


\section{INTRODUÇÃO}

A doença de Jorge Lobo (DJL), também conhecida, entre outras denominações, como lobomicose, blastomicose queloideana e lacaziose, é infecção cutânea profunda causada pelo fungo leveduriforme Lacazia loboi (L. loboi). A doença deve seu nome ao dermatologista brasileiro Jorge Lobo, que descreveu o primeiro caso em $1931 .{ }^{1}$ Os indivíduos afetados são, em sua maioria, homens trabalhadores florestais ou agricultores residentes em áreas amazônicas da América do Sul. A micose também tem sido observada em golfinhos de estuários de rios e da costa atlântica do Suriname, da Flórida, da França (um caso humano de provável fonte animal) e do estado de Santa Catarina. ${ }^{2,3,4,5}$ Os mecanismos precisos de inoculação são ainda pouco conhecidos, admitindo-se, entretanto, que a implantação traumática do fungo na pele seja a via pela qual o homem adquira a infecção. ${ }^{6,7}$ As lesões cutâneas predominam nos membros inferiores, representadas por nódulos queloideformes, tumores e placas verrucosas que, eventualmente, se ulceram. Envolvimento extensivo da pele por disseminação linfática é raramente observado. Mucosas e órgãos internos são poupados, mesmo em casos generalizados, em que se suspeita de uma rota hematogênica de disseminação.

O quadro histopatológico da $\mathrm{DJL}^{6,7,8,9,10}$, por ser bastante típico, permite um diagnóstico à primeira vista. Compreende um infiltrado granulomatoso que é em geral difuso, rico em histiócitos, com grande número de corpúsculos parasitários leveduriformes dispostos como unidades isoladas ou em agregados catenulares no citoplasma de histiócitos multinucleados. Linfócitos e plasmócitos são raros. Corpúsculos asteroides podem ser ocasionalmente observados. Os granulomas tendem a circunscrever-se à derme, envoltos por delicadas traves fibróticas. Por vezes, incluem agregados de grandes histiócitos de citoplasma claro ou eosinofílico finamente granuloso, xantomiforme, sem parasitas no citoplasma (células pseudoGaucher). A epiderme apresenta aplanamento de cristas interpapilares ou atrofia, e uma estreita faixa Grenz subepidérmica é observada com frequência. Entretanto, áreas acantóticas associadas com espongiose e coleções de neutrófilos contendo parasitas em número variável são encontradas, às vezes, o que sugere eliminação transepidérmica de parasitas. A ocorrência de ETEP na DJL também pode ser comprovada por meio do exame micológico direto, como o que utiliza fita gomada e uma solução clarificante de hidróxido de potássio a $\mathbf{1 0} \%$ em dimetil-sulfóxido a $40 \%$, quando, então, parasitas são facilmente demonstrados. Este trabalho teve por finalidade estudar aspectos morfológicos associados com ETEP na doença de Jorge Lobo.

\section{MATERIAL E MÉTODOS}

Utilizou-se material emblocado em parafina de biópsias de casos com diagnóstico de DJL disponíveis nos arquivos do laboratório de dermatopatologia do Serviço de Dermatologia do Instituto de Ciências da Saúde da Universidade Federal do Pará (ICS-UFPA). Novos cortes em cada bloco foram feitos, processados segundo a rotina e corados pela hematoxilina-eosina (H-E). O número de cortes obtidos por bloco variou segundo o permitido pelo seu estado de conservação. Agregados de parasitas de mistura com serosidade e/ou células inflamatórias dispostas sobre a camada córnea não foram interpretados microscopicamente como ETEP, mas apenas os que apresentaram indiscutível presença nas estruturas epidérmicas. Para alguns casos incluídos na amostra, apenas lâminas bem preservadas estavam disponíveis, pois os respectivos blocos haviam sido completamente esgotados em investigações anteriores. Procedeu-se a análise estatística, quando cabível, com emprego do programa Bioestat, versão 5.0.

\section{RESULTADOS}

Entraram no estudo 40 biópsias de um total de 37 pacientes (31 homens e seis mulheres, média de idade 51,03 anos, variação 29-80 anos), realizadas em um período de 37 anos (1967-2003), das quais se obtiveram e se examinaram 511 cortes (média de 12,77 cortes por caso, variação 2-39 cortes por caso). Aspectos consistentes com eliminação transepidérmica de parasitas foram observados em 110/511 cortes examinados (21,52\%) e estavam ausentes em 401/511 cortes examinados $(78,48 \%)$, sendo a diferença estatisticamente muito significante $(p<0,0001)$. Em relação aos pacientes (37), entretanto, verificaram-se, em 15 deles, aspectos consistentes com eliminação transepidérmica de parasitas (40,5\%), sendo que, em 22 dos casos $(59,5 \%)$, isso não foi observado $(p>0,05)$. Parasitas na epiderme foram detectados, invariavelmente, em infundíbulos hiperplásicos, dispostos em agregados, formando catênulas ou como unidades isoladas (Figura 1A). Havia um infiltrado inflamatório associado predominantemente neutrofílico (por vezes, constituindo microabscessos) e/ou de histiócitos e linfócitos (Figura 1B e C). Quando presentes no estrato córneo e demais estruturas epiteliais infundibulares, os parasitas eram observados tanto em crostas (Figura 1D) como não associados a células inflamatórias (Figura 2).

\section{DISCUSSÃO}

Eliminação transepidérmica (ET), originalmente descrita por Mehregan em 1970, ${ }^{12}$ representa um processo pelo qual a pele se liberta de células inflamatórias, componentes tissulares, material estranho e 

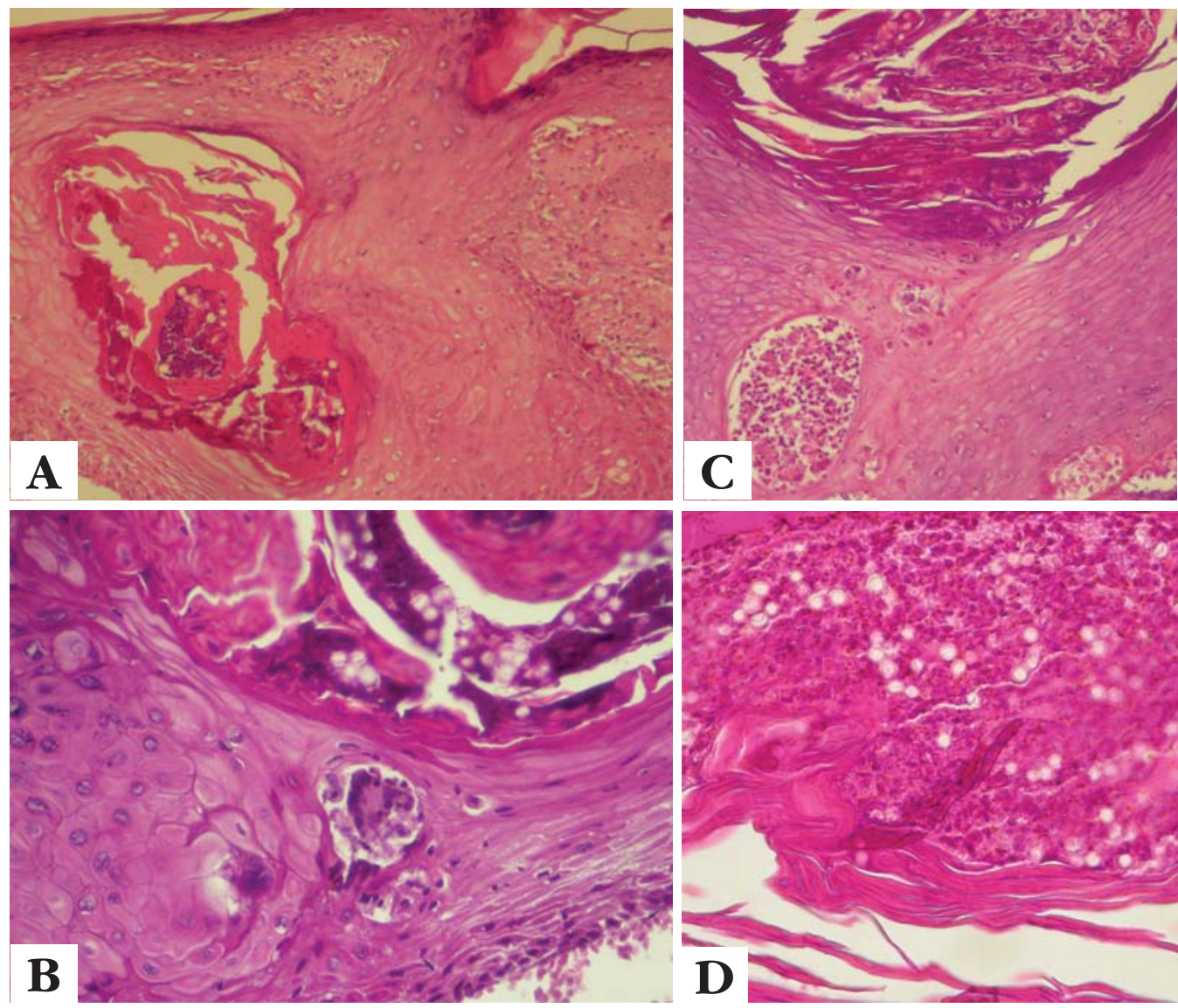

Figura 1: A. Infundíbulo hiperplásico com numerosos parasitas leveduriformes dispostos em grande tampão córneo (HE, aumento original 200x); B. Histiócitos multinucleados, linfócitos e parasitas permeiam infundíbulo (HE, aumento original 400x); C. Microabscesso e volumoso tampão córneo com incontáveis exemplares de $L$. lobo $i$ (HE, aumento original 100x); D. Agregados catenulares de $L$. loboi facilmente identificáveis numa crosta inserida em espessa camada córnea infundibular (HE, aumento original 200x)

microrganismos. Representa aspecto essencial do grupo das assim chamadas dermatoses perfurantes, tais como doença de Kyrle, colagenose perfurante reativa, elastose perfurante serpiginosa, foliculite perfurante, bem como das secundárias à insuficiência renal crônica e diabetes mellitus. ${ }^{13,14}$ A ET pode ser esporadicamente observada em condições tão diversas como granulomas não infecciosos, reações cutâneas liquenóides, deposição extracelular de substâncias, neoplasias e infecções. No que diz respeito ao último grupo, um número crescente de microrganismos vem sendo citado no contexto da ETEP, incluindo fungos, bactérias, protozoários e até mesmo clamídias. ${ }^{15}$ Parasitas dispostos em uma epiderme hiperplásica em associação com células inflamatórias, especialmente neutrófilos, formando microabscessos e/ou histiócitos, sugerindo um evento de ETEP, são especialmente observados em infecções micóticas granulomatosas e supurativas, tais como cromomicose (CM) e paracoccidioidomicose (PM) ${ }^{16,17}$ micoses profundas de maior prevalência no Brasil. Entretanto, isso não corresponde exatamente ao cenário microscópico da DJL, caracterizado por um infiltrado inflamatório granulomatoso rico em histiócitos, em que neutrófilos são apenas observados na derme superficial de lesões ulceradas. ${ }^{6,7}$ A ocorrência de ETEP na DJL foi originalmente referida por Gadelha e Bandeira em 1983, ${ }^{10}$ seguida de outras comunicações. ${ }^{8,6,7}$ Pradinaud aponta a presença de "diminutos pontos pretos" clinicamente observáveis na superfície de lesões da DJL, similares aos vistos na $\mathrm{CM}$, que corresponderiam a parasitas sendo eliminados com restos necróticos. Até onde chega o conhecimento dos autores, um estudo detalhado sobre aspectos microscópicos particulares da ETEP na DJL ainda não havia sido realizado.

\section{CONCLUSÃO}

Neste trabalho, os autores puderam demonstrar pelo menos três aspectos associados com o fenômeno de ETEP na DJL: (i) presença de uma reação exsudativa de neutrófilos, histiócitos e/ou linfócitos acompanhada de parasitas na epiderme; (ii) em outros casos, apenas corpúsculos parasitários foram observados; (iii) em ambas as situações, as alterações se processaram em infundíbulos hiperplásicos, com padrão pseudocarcinomatoso, às vezes, poupando a epiderme interinfundibular. Este último aspecto está de acordo com o conceito corrente de hiperplasia pseudocarci- 


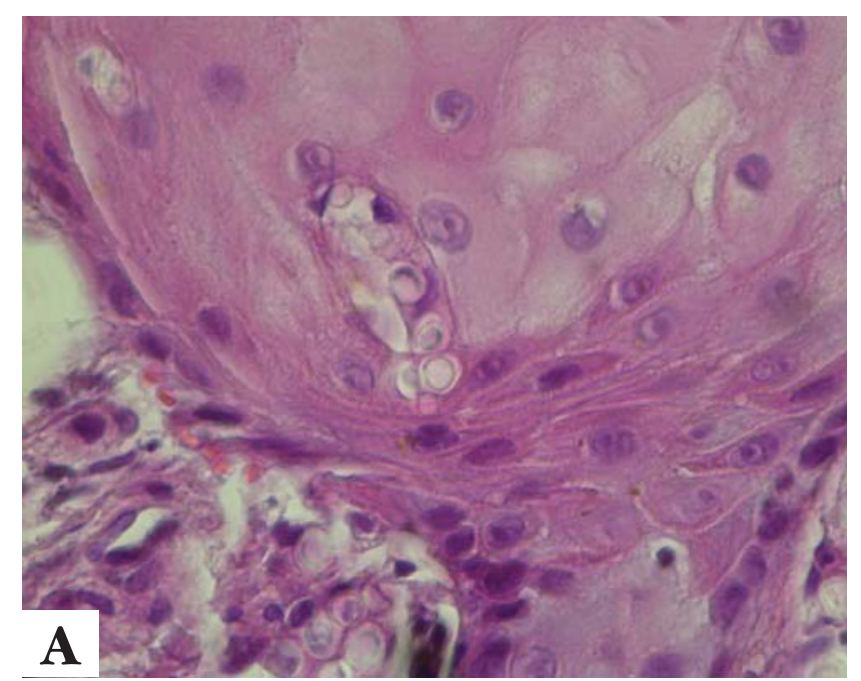

nomatosa como sendo uma proliferação do epitélio infundibular e/ou dutal écrino, que é observada em qualquer infundibulite supurativa crônica, tal como ocorre nos halogenodermas, infecções fúngicas $\mathrm{e}$ micobacterioses atípicas. Para concluir, aspectos histopatológicos consistentes com ETEP, embora obser-

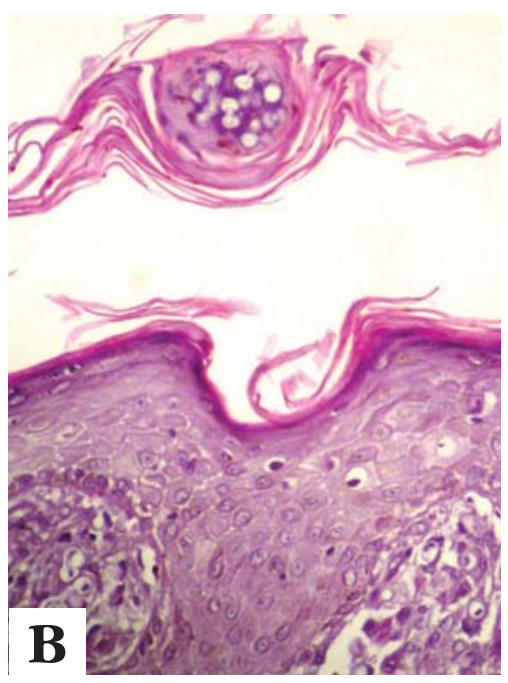

Figura 2: A. Típico dispositivo em catênula de $L$. loboi em um infundíbulo hiperplásico. Observar a ausência de células inflamatórias associadas e o infiltrado dérmico granulomatoso rico em parasitas abaixo (HE, aumento original 400x); B. Tampão córneo paraceratótico contendo parasitas (HE, aumento original $100 x)$. vados em número estatisticamente não significante de casos da amostra, sugerem que, na doença de Jorge Lobo, o fenômeno ocorra, invariavelmente, através do epitélio infundibular. Estudos futuros serão necessários para avaliar sua eventual importância na epidemiologia da micose.

\section{AGRADECIMENTOS:}

À Prof. Dra. Réia Silvia Lemos (UFPA), pelo irrestrito apoio institucional, e ao Prof. Dr. Manuel Ayres (UFPA), pela leitura crítica do manuscrito e oportunas sugestões. 


\section{REFERÊNCIAS}

1. Lobo J. Um caso de blastomicose produzido por uma espécie nova encontrada em Recife. Rev Med Pernamb. 1931;1:763-75.

2. De Vries GA, Laarman JJ. A case of Lobo's disease in the dolphin Sotalia guianensis. Aquatic Mammals. 1973;1:26-33.

3. Migaki G, Valério MG, Irvine B, Gardner FM. Lobo's disease in am Atlantic bottle-nosed dolphin. J Am Vet Med Assoc. 1971;159:578-82.

4. Symmers WSTC. A possible case of Lobo's disease acquired in Europe from a bottle-nosed dolphin (Tursiops truncatus). Bull Soc Pathol Exot Filiales 1983; 76:777-84.

5. Lopes PCS, Paula GS, Both MC, Xavier FM, Scaramello AC. First case of lobomycosis in a bottle-nose dolphin from Southern Brazil. Marine Mammal Sci. 1993;9:329-31.

6. Brito AC. Lobomycosis. In: Tyring SK, Lupi O, Hengge UR, eds. Tropical dermatology. São Paulo: Elsevier; 2006. p.207-9.

7. Brito AC, Quaresma JAS. Lacaziose (doença de Jorge Lobo): revisão e atualização. An Bras Dermatol. 2007;82:461-74.

8. Pradinaud R, Talhari S. Lobomycose. Encycl Méd Chir (Elsevier, Paris): Maladies infectieuses; 1996. p.1-6.

9. Bhawan J, Bain RW, Purtilo DT, Gomez N, Dewan C, Whelan CF, et al. Lobomycosis: an electronmicroscopic, histochemical and immunologic study. J Cutan Pathol. 1976;3:5-16.

10. Gadelha AR, Bandeira V. Micoses profundas. In: da Silva IM, ed. Dermatopatologia. Rio de Janeiro: Atheneu; 1983. p.125-34.

11. Miranda MFR, Silva AJG. Vinyl adhesive tape also effective for direct microscopy diagnosis of chromomycosis, lobomycosis, and paracoccidioidomycosis. Diagn Microbiol Infect Dis. 2005;52:39-43.
12. Mehregan AH. Transepidermal elimination. Curr Probl Dermatol. 1970; 3:124-47.

13. Woo TY, Rasmussen JE. Disorders of transepidermal elimination. Part 1. Int J Dermatol. 1985;24:267-79.

14. Woo TY, Rasmussen JE. Disorders of transepidermal elimination. Part 2. Int J Dermatol. 1985;24:337-48.

15. Ramdial PK, Kharsany AB, Reddy R, Chetty R. Transepidermal elimination of cutaneous vulval granuloma inguinale. J Cutan Pathol. 2000;27:493-9.

16. Uribe F, Zuluaga AI, León W, Restrepo A. Histopathology of chromoblastomycosis. Mycopathologia. 1989;105:1-6.

17. Uribe F, Zuluaga AI, León W, Restrepo A. Histopathology of cutanoeus and mucosal lesions in human paracoccidioidomycosis. Rev Inst Med Trop Sao Paulo. 1987; 29:90-6.

18. Ackerman AB, Chongchitnant N, Sanchez J, Guo Y, Bennin B, Reichel M, et al. Histologic diagnosis of inflammatory skin diseases. An algorithmic method based on pattern analysis. 2 ed. Baltimore: Williams \& Wilkins; 1997. p.79-80.

ENDEREÇO PARA CORRESPONDÊNCIA / MAILING ADDRESS: Mario Fernando Ribeiro de Miranda

Av. Nazaré, 1.033 / 701 - Nazaré

66035-170 Belém - PA

E-mail:mariomir@globo.com

Como citar este artigo/How to cite this article: Miranda MFR, Costa VS, Bittencourt MJS, Brito AC. Eliminação transepidérmica de parasitas na doença de Jorge Lobo. An Bras Dermatol. 2010;85(1):39-43. 